\title{
DIEUDONNÉ DETERMINANTS FOR SKEW POLYNOMIAL RINGS
}

\author{
LENNY TAELMAN
}

\begin{abstract}
We observe that the Dieudonné determinant induces a nonnegative degree function on the ring of matrices over a skew polynomial ring. We then apply this degree function to two examples. In the first one, we find an expression for the rank of the kernel of an endomorphism of the algebraic group $\mathbb{G}_{a}^{n}$ over a field of characteristic $p>0$. In the second, we calculate the dimension of the solution space of linear matrix differential equations.
\end{abstract}

\section{The Dieudonné Determinant over Skew polynomial Rings}

In this section we recall some properties of skew polynomial rings and of matrices over skew fields. We then state and prove the main result.

1.1. Skew polynomial rings. This short treatment is based on chapter 1 of Cohn 1977. Proofs can be found in there.

Let $k$ be a field endowed with an endomorphism $\alpha: k \rightarrow k: a \mapsto a^{\alpha}$ and an $\alpha$-derivation $\delta$. The latter is an additive map $\delta: k \rightarrow k: a \mapsto a^{\delta}$ such that

$$
(a b)^{\delta}=a^{\delta} b^{\alpha}+a b^{\delta} .
$$

If $\alpha=1$, this is just a derivation. The left skew polynomial ring in $x$ over $k$ associated with $\alpha$ and $\delta$, denoted by $R=k[x ; \alpha, \delta]$ is the ring whose elements are expressions of the form $a_{0}+a_{1} x+\ldots+a_{m} x^{m}$ with $a_{i} \in k$. Addition is defined in the usual way. Multiplication is defined by the commutation rule

$$
x a=a^{\alpha} x+a^{\delta}
$$

The left euclidean algorithm holds in $R$, relative to the usual degree map deg : $R-\{0\} \rightarrow \mathbb{N}$. Hence, $R$ is a left principal ideal domain. When $\alpha$ is an automorphism of the field $k$ then $R$ is also a right skew polynomial ring and hence also right euclidean.

Since $R$ is a left principal ideal domain, it is left Ore and it has a unique skew field of fractions $Q$. It consists of expressions of the form $r / s$ with $s \neq 0$ and $r / s=r^{\prime} / s^{\prime}$ if and only if there exist non-zero $u, u^{\prime}$ in $R$ with $u r=u^{\prime} r^{\prime}$ and $u s=u^{\prime} s^{\prime}$. The degree map $\operatorname{deg}: Q \rightarrow \mathbb{Z} \cup\{\infty\}: r / s \mapsto \operatorname{deg}(r)-\operatorname{deg}(s)$ is well-defined and satisfies

$$
\operatorname{deg}(t u)=\operatorname{deg}(t)+\operatorname{deg}(u) .
$$

It factors over $Q \rightarrow Q^{\mathrm{ab}}=Q^{\times} /\left[Q^{\times}, Q^{\times}\right] \cup\{0\}$.

1.2. Matrices over skew polynomial rings. $\mathrm{M}(n, S)$ is the ring of $n \times n$ matrices over the ring $S$. The subring of diagonal matrices is denoted by $\mathrm{D}(n, S)=S^{n}$. We write $\mathrm{GL}(n, S)$ for the group of invertible elements in $\mathrm{M}(n, S)$ and $\mathrm{E}(n, S)$ for the subgroup generated by the elementary matrices (corresponding to the elementary row and column operations). 
Consider the ring $\mathrm{M}(n, Q)$ and its subring $\mathrm{M}(n, R)$. On the former, the Dieudonné determinant (Dieudonné 1943, Draxl 1983) defines a multiplicative function

$$
\operatorname{det}: \mathrm{M}(n, Q) \rightarrow Q^{\mathrm{ab}}
$$

and an exact sequence of groups

$$
1 \rightarrow \mathrm{E}(n, Q) \rightarrow \mathrm{GL}(n, Q) \rightarrow\left(Q^{\mathrm{ab}}\right)^{\times} \rightarrow 1
$$

Furthermore, $\mathrm{E}(n, Q)$ equals the commutator subgroup of $\mathrm{GL}(n, Q) \cdot{ }^{1}$ As the function det is rational in the entries of the matrix, we cannot hope for a restriction to $\mathrm{M}(n, R)$ with the same properties as its commutative, polynomial, counterpart. However, consider the map

$$
\operatorname{deg} \operatorname{det}: \mathrm{M}(n, Q) \rightarrow \mathbb{Z} \cup\{\infty\} .
$$

It is surjective and satisfies $\operatorname{deg} \operatorname{det}(A B)=\operatorname{deg} \operatorname{det}(A)+\operatorname{deg} \operatorname{det}(B)$. We make a little observation on its restriction to $\mathrm{M}(n, R)$.

Theorem 1.1. The restriction of the map deg det to $\mathrm{M}(n, R)$ induces an exact sequence of unitary semigroups

$$
1 \rightarrow \mathrm{GL}(n, R) \rightarrow \mathrm{M}(n, R) \rightarrow \mathbb{N} \cup\{\infty\} \rightarrow 0 .
$$

Moreover, deg det is unique in the sense that every semigroup homomorphism $f$ : $\mathrm{M}(n, R) \rightarrow \mathbb{Z} \cup\{\infty\}$ that coincides with deg det on $\mathrm{D}(n, R)$ equals deg det.

Proof. We first prove the uniqueness claim and then use it to deduce the first part of the theorem.

Step 1. Assume $f: \mathrm{M}(n, R) \rightarrow \mathbb{Z} \cup\{\infty\}$ extends to $\mathrm{M}(n, Q)$. As deg has a unique extension from $R$ to $Q$, we find that $f=\operatorname{deg} \operatorname{det}$ on $\mathrm{D}(n, Q)$. Since $\mathrm{M}(n, Q)=$ $\mathrm{D}(n, Q) \mathrm{M}(n, R)$, there can at most be one extension of $f$. It is a straightforward computation to show that the extension given by $f(D A)=f(D)+f(A)$ with $D \in \mathrm{D}(n, Q)$ and $A \in \mathrm{M}(n, R)$ is a well-defined semigroup homomorphism. Observe that this unique lift $f: \mathrm{M}(n, Q) \rightarrow \mathbb{Z} \cup\{\infty\}$ has to factor over det $: \mathrm{M}(n, Q) \rightarrow Q^{\text {ab }}$. Because $\mathrm{D}(n, Q)$ maps surjectively to $Q^{\text {ab }}$ and $f$ coincides with $\operatorname{deg} \operatorname{det}$ on $\mathrm{D}(n, Q)$, we conclude that $f=\operatorname{deg} \operatorname{det}$.

Step 2. Given a matrix $A \in \mathrm{M}(n, R)$, consider the quotient of left $R$-modules $R^{n} / R^{n} A$. It is a vector space over $k$. Now define

$$
f: \mathrm{M}(n, R) \rightarrow \mathbb{N} \cup\{\infty\}: A \mapsto \operatorname{dim}_{k} R^{n} / R^{n} A .
$$

Because $f(A B)=f(A)+f(B)$ and because $f(D)=\operatorname{deg} \operatorname{det}(D)$ for diagonal matrices $D$, we conclude that $f=\operatorname{deg} \operatorname{det}$ on all of $\mathrm{M}(n, R)$. But $f$ takes values in $\mathbb{N} \cup\{\infty\}$ and $\operatorname{ker} f=\operatorname{GL}(n, R)$. This finishes the proof.

\section{Applications}

By interpreting the skew polynomial ring, we can apply the main result to two special cases. The first has a trivial derivation, the second a trivial field endomorphism.

\footnotetext{
${ }^{1}$ Over every skew field, $\mathrm{E}$ is the kernel of det. It equals the commutator subgroup of GL unless $n=2$ and the skew field is the finite field of two elements.
} 
2.1. Endomorphisms of $\mathbb{G}_{a}^{n}$ in characteristic $p>0$. Let $k$ be a field of characteristic $p>0$. Denote by $\mathbb{G}_{a}$ the additive (algebraic) group over $k$. Its ring of endomorphisms is known to be the skew polynomial ring $R=k\left[\tau ; a \mapsto a^{p}, 0\right]$. Here $a \in k$ acts on $\mathbb{G}_{a}$ by multiplication with $a$ and $\tau$ acts as the $p$-th power frobenius. More generally, we have a canonical isomorphism

$$
\operatorname{End}\left(\mathbb{G}_{a}^{n}\right)=\mathrm{M}(n, R)
$$

Given a $\phi \in \operatorname{End}\left(\mathbb{G}_{a}^{n}\right)$, its kernel is a closed subgroup scheme with an $\mathbb{F}_{p}$-linear structure. We can now determine its size.

Corollary 2.1. If $\operatorname{deg} \operatorname{det} \phi<\infty$ then $\operatorname{ker} \phi$ is a closed subgroup scheme of $\mathbb{G}_{a}^{n}$ which is finite of rank $p^{\operatorname{deg} \operatorname{det} \phi}$ over $\operatorname{Spec}(k)$.

Proof. Consider the function $f: \operatorname{End}\left(\mathbb{G}_{a}^{n}\right) \rightarrow p^{\mathbb{N}} \cup\{\infty\}$ that associates with $\phi$ the rank of ker $\phi$ whenever this kernel is finite and $\infty$ when it is not. One readily checks that $f$ is multiplicative and that $f$ coincides with $p^{\operatorname{deg} \operatorname{det}}$ for diagonal $\phi$. The result now follows from theorem 1.1

Remark 2.2 (Anderson abelian $t$-modules). In the theory of Anderson $t$-motives (Anderson 1986) one considers 2 types of objects. They roughly look as follows. The first object is an abelian t-module of dimension $n$ and is an action of $\mathbb{F}_{p}[t]$ on $\mathbb{G}_{a}^{n} / k$. The second object, called a $t$-motive of dimension $n$ and rank $r$ is a module over $R \otimes_{\mathbb{F}_{p}} \mathbb{F}_{p}[t]$, which has rank $n$ over $R=k\left[\tau ; a \mapsto a^{p}\right]$ and rank $r$ over $k \otimes \mathbb{F}_{p}[t]$. The motives are in some sense dual to the modules and one proves that these objects actually form anti-equivalent categories. After this has been proven, one defines the rank of an abelian $t$-module to be the rank of its associated $t$-motive.

However, no straightforward description of the rank of an abelian $t$-module, other than the rank of its associated motive, seemed to be known for $n>1$. When $n=1$ we are dealing with Drinfel'd modules and a very simple description in terms of the degree of the endomorphisms acting on $\mathbb{G}_{a}$ is known. Corollary 2.1 shows that deg det is the sought generalization of this degree function and gives the following desctiption. The rank of the abelian $t$-module given by

$$
\phi: \mathbb{F}_{p}[t] \rightarrow \operatorname{End}\left(\mathbb{G}_{a}^{n} / k\right)=\mathrm{M}(n, R),
$$

is the constant $r \in \mathbb{N}$ which satisfies

$$
\left(\forall f \in \mathbb{F}_{p}[t]\right)(\operatorname{deg} \operatorname{det} \phi(f)=r \operatorname{deg} f) .
$$

2.2. Systems of linear ordinary differential equations. Details on the algebraic theory of differential equations can be found in the book van der Put - Singer 2003 .

Let $k$ be a differential field of characteristic 0 . This means $k$ is equipped with an additive map (called derivation) $k \rightarrow k: a \mapsto a^{\prime}$ satisfying $(a b)^{\prime}=a^{\prime} b+a b^{\prime}$. Let $C \subset k$ be the field of constants, that is the kernel of the derivation. Assume that the derivation is non-trivial, i.e. $C \neq k$. Examples are $k=\mathbb{C}(x)$ and $k=\mathbb{C}((x))$ with the usual derivation. Now consider the skew polynomial ring $R=k\left[\partial ; 1,{ }^{\prime}\right]$ with center $C$. It acts $k$-linearly on differential field extensions of $k$ by $\partial a=a^{\prime}$.

A homogeneous matrix differential equation of the form

$$
A_{0} y+A_{1} y^{\prime}+\ldots+A_{d} y^{(d)}=0
$$


where $y$ denotes a vector in $k^{n}$ and the $A_{i}$ are matrices in $\mathrm{M}(n, k)$ can be rewritten as

$$
A y=0
$$

with $A=\sum A_{i} \partial^{i} \in \mathrm{M}(n, R)$. Conversely, every $A$ corresponds to such a differential equation. Note that we do not demand $A_{d}$ to be invertible.

As in the proof of theorem 1.1] we associate with $A$ the $R$-module

$$
M:=R^{n} / R^{n} A=R^{s} \oplus M_{\text {tors }}
$$

Take $k \subset l$ to be the Picard-Vessiot extension of $M_{\text {tors }}$, or alternatively, take $l$ to be a universal differential field extension of $k$. When $s=0$, all solutions of the differential equation exist over $l$. The contravariant solution space $V$ of $M$ is defined to be the $C$-vector space

$$
V:=\operatorname{Hom}_{R}(M, l) .
$$

It is finite-dimensional if and only if $s=0$, and in that case it is dual to the $C$-space of solutions in $l^{n}$ of the given differential equation. On its dimension, we conclude:

Corollary 2.3. With the notations as above, we have

$$
\operatorname{dim}_{C} V=\operatorname{dim}_{k} M=\operatorname{deg} \operatorname{det} A .
$$

Remark 2.4. Completely analogous results hold for difference and $q$-difference equations.

\section{REFERENCES}

[Anderson 1986] G. W. Anderson. t-motives. Duke Math. J., 53(2):457-502, 1986.

[Cohn 1977] P. M. Cohn. Skew Field Constructions. Cambridge University Press, Cambridge, 1977. London Mathematical Society Lecture Note Series, No. 27.

[Dieudonné 1943] J. Dieudonné. Les déterminants sur un corps non commutatif. Bull. Soc. Math. France, 71:27-45, 1943.

[Draxl 1983] P. K. Draxl. Skew Fields. Cambridge University Press, Cambridge, 1983. London Mathematical Society Lecture Note Series, No. 81.

[van der Put - Singer 2003] M. van der Put, F. Singer. Galois Theory of Linear Differential Equations. Springer-Verlag, Berlin Heiderlberg, 2003. Grundlehren der mathematischen Wissenschaften, No. 328.

E-mail address: lenny@math.rug.nl

iWi RuG, Postbus 800, 9700AV Groningen, Nederland 